\title{
Glass Fiber Poling by an Extended Cavity Microchip Laser
}

\author{
U. Minoni ${ }^{1}$, G. Treccani ${ }^{1}$, A. Tonello ${ }^{2}$, K. Krupa ${ }^{1}$, D. Modotto ${ }^{1, *}$, S. Wabnitz ${ }^{1,3}$, V. Couderc ${ }^{1}$ \\ ${ }^{I}$ Dipartimento di Ingegneria dell'Informazione, Università di Brescia, via Branze 38, 25123 Brescia, Italy \\ ${ }^{2}$ Université de Limoges, XLIM, UMR CNRS 7252,123 Av. A. Thomas, 87060 Limoges, France \\ ${ }^{3}$ Istituto Nazionale di Ottica del Consiglio Nazionale delle Ricerche (INO-CNR), via Branze 45, 25123 Brescia, Italy \\ ${ }^{*}$ Corresponding author: daniele.modotto@unibs.it
}

\begin{abstract}
We experimentally prove that glass fibers are efficiently optically poled when inserted in the extended cavity of a microchip laser. Methodical second harmonic measurements confirm the good quality of the poling.

OCIS codes: (060.4370) Nonlinear optics, fibers; (190.2620) Harmonic generation and mixing; (140.3515) Lasers, frequency doubled; (140.4780) Optical resonators.
\end{abstract}

\section{Introduction}

Optical poling can turn a glass optical fiber in a versatile waveguide exhibiting both quadratic and cubic nonlinearities. The complex photoinduced processes leading to the growth of the quadratic coefficient $\chi^{(2)}$ require sufficiently high peak power pump pulses, and a few hours of writing time [1-3]. The poling mechanism introduces a periodic $\chi^{(2)}$ which guarantees the quasi-phase matching condition, thus enabling the poled fiber to be used to efficiently generate the second harmonic ( $\mathrm{SH}$ ) of a laser emitting at the same wavelength of the laser used to pole the fiber. The above-mentioned constraint on the laser power has so far prevented the large-scale diffusion of the optical poling, despite its simplicity.

Pulsed microchip lasers are commonly used in the nonlinear optics community. However, these lasers are inadequate to start the fiber poling process, owing to their relatively low peak power. In this work, we demonstrate a new method which permits the use of microchip lasers for fiber poling. The method involves the insertion of the fiber to be poled into an extended cavity of the microchip laser. The composite cavity arrangement leads to a resonant power enhancement of the recirculating pulses, which enables fiber poling.

\section{Fiber poling}

In our experiments, we used a commercial HI980 Corning fiber with cut-off wavelength at $0.93 \mu \mathrm{m}$, which makes it well suited for poling at $1064 \mathrm{~nm}$. The microchip Nd:YAG laser used for both poling and subsequent SH measurements emits $800 \mathrm{ps}$ pulses with the repetition frequency of about $7.1 \mathrm{kHz}$, and average power around $100 \mathrm{~mW}$. Based on the measured fiber coupling efficiency of about 50\%, the pulses injected into the fiber had a peak power lower than $10 \mathrm{~kW}$, which is the minimum value required to pole the HI980 fiber [4].

We built an extended cavity by using a lens and a mirror to feedback the laser output into the main microchip laser cavity. By carefully positioning the lens and the mirror, the extended cavity becomes stable. In this condition, we measured an average power of $390 \mathrm{~mW}$ inside the extended cavity. The almost 4-fold power increase is due to the fact that the extended cavity allows for a more efficient use of the gain of the active material inside the microchip. Figure 1 shows the setup used to build the extended cavity, including the optical fiber to be poled. The extended cavity is bounded by the rear laser mirror RM of the microchip and by partially reflecting mirror PRM with $95 \%$ reflectivity.

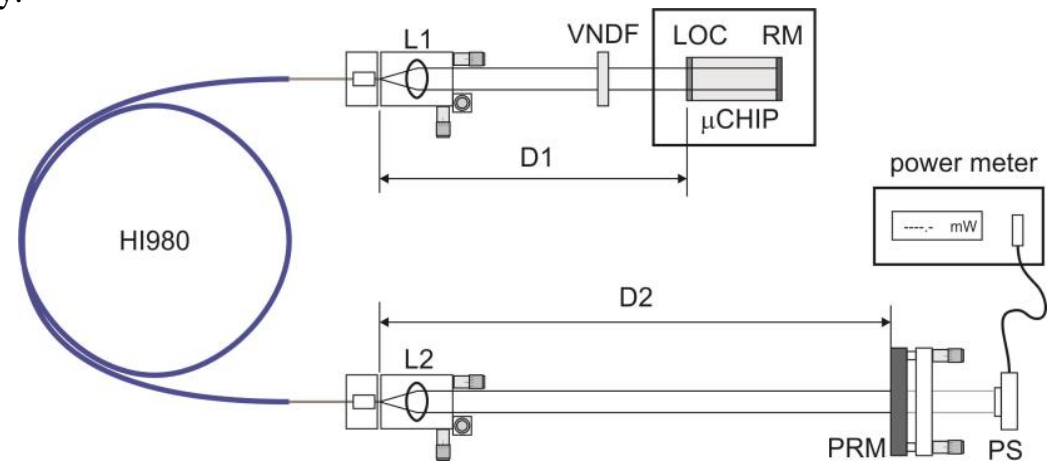

Fig. 1. Experimental setup used to pole the $1.7 \mathrm{~m}$ long HI980 fiber inside the extended cavity. RM: microchip rear mirror; LOC: laser output coupler; VNDF variable neutral density filter; PRM: partially reflecting mirror; L1, L2: micro-lenses; PS: optical power sensor. 
The demanding task of aligning the external cavity was accomplished at low powers, controlled by the variable neutral density filter VNDF, and measured through the power sensor PS which was placed behind the PRM. The distance D2 between the PRM and the fiber output was carefully adjusted, in order to optimize the resonance of the extended cavity. An average power of $230 \mathrm{~mW}$ was measured in the section of extended cavity containing the fiber. This power is about $40 \%$ lower than that measured in the extended cavity without the fiber, which can be explained by considering the significant insertion losses of the fiber and, mainly, of the components required for its insertion into the cavity. After accurate alignment and with the pump power raised to its maximum value, soon a SH beam at $532 \mathrm{~nm}$ became visible at the fiber output. After about 4 hours, the green light intensity reached a maximum value, indicating saturation of the poling mechanism.

\section{Second harmonic characterization}

The setup of Fig. 1, modified by removing the PRM, was used to measure the SH power $\mathrm{P}_{\mathrm{SH}}$ generated inside the poled fiber, as a function of the fundamental power $\mathrm{P}_{\mathrm{FF}}$. Figure 2(a) shows, on a log-log scale, that $\mathrm{P}_{\mathrm{SH}}$ depends on the square of $\mathrm{P}_{\mathrm{FF}}$. Since the microchip laser emits a linearly polarized beam, the $\mathrm{SH}$ conversion efficiency is at its maximum when the fundamental polarization coincides with that used during the poling process. After inserting a rotating half-wave plate at the laser output, we measured $\mathrm{P}_{\mathrm{SH}}$ as a function of the orientation of the input linear polarization for a fixed $\mathrm{P}_{\mathrm{FF}}$ equal to $2.5 \mathrm{~mW}$. The results are reported in Fig. 2(b). As expected, the maximum conversion efficiency is obtained when the pump laser has the same polarization orientation as that used for poling.
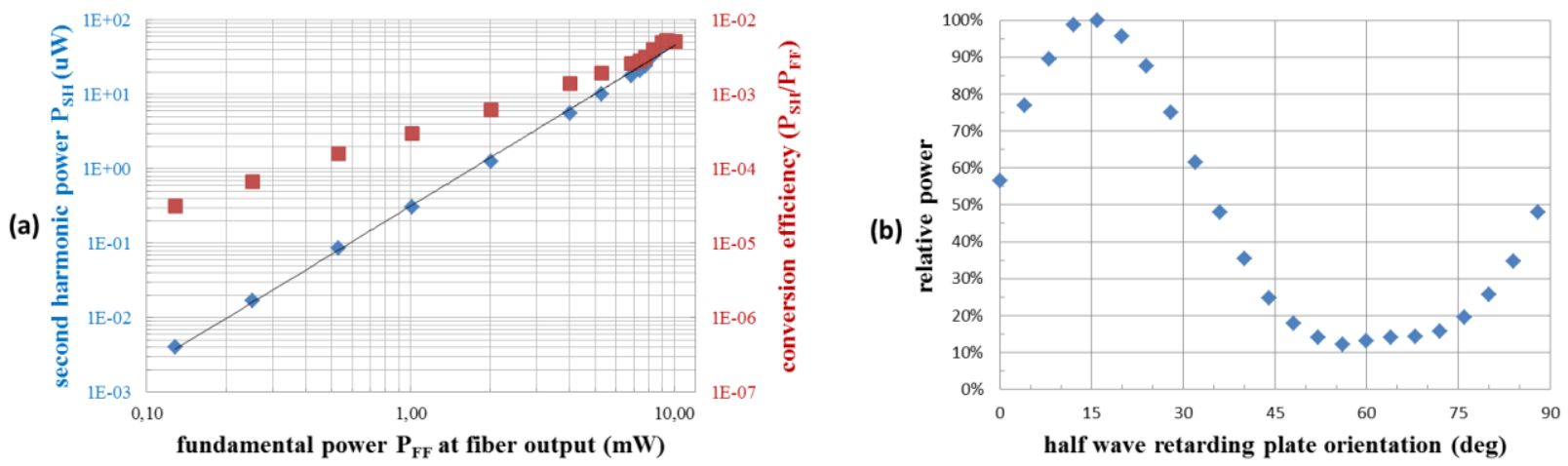

Fig. 2. (a) Measured output average SH power vs output average fundamental power; (b) normalized SH power vs the angular position of the half-wave retarding plates, $15 \mathrm{deg}$ corresponds to the polarization orientation used for poling.

\section{Conclusions}

We demonstrated that, by using a composite cavity setup, a relatively low power microchip laser can effectively be used to pole an optical fiber. Since the proposed poling technique relies on low-cost microchip lasers and standard optical components, we expect that it will be widely adopted for efficiently writing a quadratic nonlinearity in optical fibers.

\section{Acknowledgements}

We acknowledge support from: the European Research Council (ERC) under the European Union's Horizon 2020 research and innovation programme, grant No. 740355 and Marie-Sklodowska-Curie grant No. GA-2015-713694 (MULTIPLY); Horiba Medical and Leukos (MSCA MULTIPLY, BECLEAN project).

\section{References}

[1] U. Österberg and W. Margulis, "Experimental studies on efficient frequency doubling in glass optical fibers," Opt. Lett. 12, 57-59 (1987).

[2] E.M. Dianov and D.S. Starodubov, "Photoinduced generation of the second harmonic in centrosymmetric media," Sov. J. Quantum Electron. 25, 395-407 (1995).

[3] V. Couderc, A. Tonello, C. Buy-Lesvigne, P. Leproux, and L. Grossard, "Unprecedented Raman cascading and four-wave mixing from second-harmonic generation in optical fiber," Opt. Lett. 35, 145-147 (2010).

[4] D. Ceoldo, U. Minoni, D. Modotto, B.M. Shalaby, K. Krupa, A. Tonello, and V. Couderc, "Second harmonic generation in an optically poled fiber", Spatiotemporal Complexity in Nonlinear Optics (SCNO), Como, Italy, 31 Aug.-4 Sept. 2015, Article number 7324035, DOI: 10.1109/SCNO.2015.7324035. 